\title{
Geometric construction of the quantum Hall effect in all even dimensions
}

\author{
Guowu Meng \\ Department of Mathematics, Hong Kong University of Science and Technology, \\ Clear Water Bay, Kowloon, Hong Kong, People's Republic of China \\ E-mail: mameng@ust.hk
}

Received 24 April 2003, in final form 27 June 2003

Published 27 August 2003

Online at stacks.iop.org/JPhysA/36/9415

\begin{abstract}
The quantum Hall effects in all even dimensions are uniformly constructed. Contrary to some recent accounts in the literature, the existence of quantum Hall effects (QHE) does not crucially depend on the existence of division algebras. For QHE on flat space of even dimensions, both the Hamiltonians and the ground-state wavefunctions for a single particle are explicitly described. This explicit description immediately tells us that QHE on a higher even-dimensional flat space shares common features such as incompressibility with QHE on a plane.
\end{abstract}

PACS numbers: 73.43.-f, 02.20.-a, 02.40.-k, 03.65.-w

\section{Introduction}

Recently there has been a flurry on a generalization of the quantum Hall effect (QHE) to four-dimensional flat space [1-10], inspired by a paper [11] by Zhang and Hu. In view of its significance to condensed matter physics and to fundamental physics (see the conclusion and the introduction in [11]), it is worth the effort to make a closer examination of this generalization of the QHE.

In their search for QHE on four-space (i.e., $\mathbb{R}^{4}$ ), Zhang and Hu follow Haldane's approach to the QHE problem in [12]. There are two steps in this approach: (1) study the quantum mechanics problem of a single charged particle under the influence of a natural background magnetic field of strength $I$ on the sphere of radius $R$ and (2) map the sphere to the flat Euclidean space by standard stereographical mapping and then take the thermodynamic limit as both $I$ and $R$ go to infinity while keeping $I / R^{2}$ constant to recover QHE on the plane. Put it differently, QHE on the two-sphere devised by Haldane is just a family of spherical approximations to QHE on two-space (i.e., $\mathbb{R}^{2}$ ), and the strategy adopted in [11] is to generalize the spherical models of Haldane to dimension four (using an earlier work of Yang [13]) and then take the thermodynamic limit to obtain QHE on four-space. 
As a matter of fact, the natural generalization of QHE on the two-sphere goes beyond dimension four. This observation was also independently made (but not carried out) by Fabinger in [9] from the point of view of fuzzy spheres [14].

It is well known that, to understand the QHE, a key step is to understand the Hamiltonian and the ground-state wavefunctions for a single particle on flat space. However, to our best understanding of the references cited above, this very important question for QHE on fourspace has not been explicitly addressed so far. Our answer to this question for QHE on higher dimensional flat spaces turns out to be rather simple mathematically. This simplicity immediately tells us that QHE on higher dimensional flat space shares common features such as incompressibility with QHE on two-space.

\section{QHE on even-dimensional spheres}

Here we formulate the quantum mechanics model ${ }^{1}$ for the QHE problem on even-spheres in clean geometric language. The approach in [11], where the ground-state wavefunctions are given first and the Hamiltonian is derived later, while it works in dimension four, does not seem to work in higher even dimensions. Our approach starts with the Hamiltonian and is more straightforward and works in any even dimension. However, the discussion here may not be really new in a broad sense, and it could have been known a long time ago to mathematicians in the context of representation theory of compact Lie groups. In any case, similar discussions in more general settings appeared (partially or fully) in the mathematical physics literature repeatedly in the past [16-18].

Following [11], a point $X_{i}$ on $\mathrm{S}^{2 n}(R)$ (the $2 n$ sphere centred at the origin with radius $R$ ) can be described by dimensionless vector coordinates $x_{i}=X_{i} / R$, with $i=1,2, \ldots, 2 n+1$, which satisfy $x_{i}^{2}=1$. Now, $\mathrm{S}^{2 n} \equiv \mathrm{S}^{2 n}(1)$ is the homogeneous space $\operatorname{spin}(2 n+1) / \operatorname{spin}(2 n)$, and the principal bundle

$$
\operatorname{spin}(2 n) \rightarrow \operatorname{spin}(2 n+1) \rightarrow \mathrm{S}^{2 n}
$$

has a canonical connection

$$
A=\operatorname{Pr}_{s o(2 n)}\left(g^{-1} d g\right)
$$

where $g^{-1} d g$ is the Cartan-Maurer form on $\operatorname{spin}(2 n+1)$ and $\operatorname{Pr}_{s o(2 n)}$ is the orthogonal projection onto the Lie algebra of $\operatorname{spin}(2 n)$. Let $\Delta$ be the fundamental spin representation of $\operatorname{spin}(2 n+1)$, then $\Delta=\Delta^{+} \oplus \Delta^{-}$as representations of $\operatorname{spin}(2 n)$, where $\Delta^{ \pm}$are the positive/negative spin representations of $\operatorname{spin}(2 n)$. The highest weight state of $\Delta^{ \pm}$is $|\underbrace{\frac{1}{2} \cdots \frac{1}{2}}_{n-1} \frac{ \pm 1}{2}\rangle$. Let $I$ be a positive half integer, $\Delta_{I}^{+}$be the irreducible representation of $\operatorname{spin}(2 n)$ with highest weight state $|\underbrace{I \cdots I}_{n}\rangle$. (In general $\Delta_{I}^{+}$is an irreducible component of the $2 I$-fold symmetric tensor product of $\Delta^{+}$.) Form the complex vector bundle $\xi_{I}$ : $\operatorname{spin}(2 n+1) \times_{\operatorname{spin}(2 n)} \Delta_{I}^{+} \rightarrow S^{2 n}$, then $\xi_{I}$ has an induced canonical $\operatorname{spin}(2 n)$-connection $A_{I}$.

1 Before going into the mathematical details, I would like to point out that, physically, it is the quantum mechanics of a charged particle in a $2 n$-dimensional sphere under the influence of a canonical background spin( $2 n)$-gauge field. For further explanation, consult appendix A. 
The quantum mechanics problem is the study of a charged particle in the presence of background magnetic potential $A_{I}$, so the Hamiltonian ${ }^{2}$ is

$$
\hat{H}=\frac{\hbar^{2}}{2 M R^{2}} d_{A_{I}}^{\dagger} d_{A_{I}}
$$

where $d_{A_{I}}$ is the covariant derivative: $\Gamma\left(\xi_{I}\right) \rightarrow \Gamma\left(\xi_{I} \otimes T^{*} S^{2 n}\right)$, and $d_{A_{I}}^{\dagger}$ is the formal adjoint of $d_{A_{I}}$.

To compute the spectrum of $\hat{H}$ in equation (3), we note that

$$
d_{A_{I}}^{\dagger} d_{A_{I}}=c_{2}(\operatorname{spin}(2 n+1))-c_{2}\left(\operatorname{spin}(2 n), \Delta_{I}^{+}\right)
$$

where $c_{2}(\operatorname{spin}(2 n+1))$ is the quadratic Casimir operator of $\operatorname{spin}(2 n+1)$ and $c_{2}\left(\operatorname{spin}(2 n), \Delta_{I}^{+}\right)$ is the value of the quadratic Casimir operator of $\operatorname{spin}(2 n)$ on $\Delta_{I}^{+}$. Therefore we have

$$
\hat{H}=\frac{\hbar^{2}}{2 M R^{2}}\left[c_{2}(\operatorname{spin}(2 n+1))-c_{2}\left(\operatorname{spin}(2 n), \Delta_{I}^{+}\right)\right] .
$$

Note that equations (3) and (5) have appeared in [16-18] in a slightly different form (see appendix A for more details).

The Hilbert space of this quantum system is the space of square integrable sections of $\xi_{I}$ and it decomposes into the direct sum of the eigenspaces of $\hat{H}$. These energy eigenspaces, indexed by integer $q \geqslant 0$, are all irreducible representation spaces of $\operatorname{spin}(2 n+1)$. The $q$ th energy eigenspace $\mathcal{H}_{I}(q)$ is labelled by its highest weight state $|(q+I) \underbrace{I \cdots I}_{n-1}\rangle$, and the corresponding eigenvalue is $E(q)=\frac{\hbar^{2}}{2 M R^{2}}\left[2 I\left(q+\frac{n}{2}\right)+q(q+2 n-1)\right]$. The ground state, which is the lowest $\operatorname{spin}(2 n+1)$ level for a given $I$, is obtained by setting $q=0$, and is $d_{0} \equiv \prod_{1 \leqslant i \leqslant j \leqslant n}\left(1+\frac{2 I}{2 n+1-i-j}\right)$-fold degenerate. (All these are standard results in mathematics and can be found in a textbook on group representations, for example [19].) Therefore, I plays the role of magnetic flux, while $q$ plays the role of the Landau level index. States with $q>0$ are separated from the ground state by a finite energy gap. Note that in the limit $I \rightarrow \infty$ and $R \rightarrow \infty$ while keeping $l_{0} \equiv \frac{R}{\sqrt{2 I}}$ and $q$ constant,

$$
E(q) \rightarrow \frac{\hbar^{2}}{2 M l_{0}^{2}}\left(q+\frac{n}{2}\right)
$$

and the single particle energy spacing is finite. This can also be seen from the thermodynamic limit of $\hat{H}$ below.

\section{QHE on even-dimensional flat spaces}

Here we shall see that $\hat{H}$ in equation (3) is rather simple in the thermodynamic limit. As a first step, we shall find the expression for $\hat{H}$ on $S^{2 n} \backslash\{S\}$ (i.e., the sphere with the south pole $S$ removed). To do this, we need to fix a gauge on $S^{2 n} \backslash\{S\}$, i.e., a smooth section $\phi$ on $S^{2 n} \backslash\{S\}$ of the principal bundle in (1). We prefer to choose an $S O(2 n)$-equivariant gauge, for example, we may take

$$
\text { Landau gauge: } \phi(\vec{y})=\left(\begin{array}{cc}
I-\frac{2 \vec{y} \vec{y}^{T}}{1+y^{2}} & \frac{2 \vec{y}}{1+y^{2}} \\
-\frac{2 \vec{y}^{T}}{1+y^{2}} & \frac{1-y^{2}}{1+y^{2}}
\end{array}\right)
$$

${ }^{2}$ Here is a remark for QHE on a four-sphere. Note that spin $(4)=S U(2) \times S U(2)$, so the background gauge field splits into two components. However, since the particle is neutral with respect to the second component gauge field, physically, the particle only sees $S U(2)$ - a component of $\operatorname{spin}(4)$. So the effective principal bundle used here is just a $S U$ (2) bundle which can be seen to be precisely the Hopf bundle used in [11]. (These observations have already appeared in a series of papers by Y S Wu and his collaborators which were published in some Chinese journals in the 1970s.) The advantage of our construction is that the spin(5) (not $S O(5)$ ) symmetry of the system is manifest from the very beginning. Compare with [11]. 
where $\vec{y}$ is the standard stereographical map $S^{2 n} \backslash\{S\} \rightarrow \mathbb{R}^{2 n}$, viewed as a coordinate map. Then the connection form on $S^{2 n} \backslash\{S\}$ under the Landau gauge is

$$
\phi^{*}(A)=2\left(\vec{y} d \vec{y}^{T}-d \vec{y} \vec{y}^{T}\right)+O\left(|\vec{y}|^{3}\right)
$$

where $O\left(|\vec{y}|^{3}\right)$ is a term whose coefficient in each $d y^{i}$ is of order $|\vec{y}|^{3}$ as $\vec{y} \rightarrow \overrightarrow{0}$.

For the purpose of taking limits, it is convenient to have another representation of $S O(2 n)$ [19]: a complex $2 n \times 2 n$ matrix $X$ is in $S O(2 n)$ if and only if it is anti-Hermitian and $J X^{T} J+X=0$, where $J=\left(\begin{array}{cc}0 & I_{n} \\ I_{n} & 0\end{array}\right)$ with $I_{n}$ being the $n \times n$ identity matrix. In this representation, the natural connection form in equation (8) becomes

$$
B=\left(\frac{z d z^{\dagger}-d z z^{\dagger}}{z d z^{T}-d z z^{T}} \quad \frac{z d z^{T}-d z z^{T}}{z d z^{\dagger}-d z z^{\dagger}}\right)+O\left(|z|^{3}\right)
$$

where $z=\left(\begin{array}{c}z^{1} \\ \vdots \\ z^{n}\end{array}\right)$ with $z^{\mu}=y^{\mu}+\sqrt{-1} y^{n+\mu}$ for $1 \leqslant \mu \leqslant n$ and a bar means complex conjugation. Then the Hamiltonian operator (in the Landau gauge) is

$$
\hat{H}=-\frac{1}{2 M R^{2}} \frac{1}{\sqrt{h}}\left(\partial_{j}+B_{j}\right)\left[h^{j k} \sqrt{h}\left(\partial_{k}+B_{k}\right)\right]
$$

where $h$ is the following Riemannian metric on $\mathbb{C}^{n}=\mathbb{R}^{2 n}$ :

$$
h_{i j}(\vec{y})=\frac{c}{1+\vec{y}^{2}} \delta_{i j}
$$

where $c$ is a constant which can/will be set to be 1 .

To find the thermodynamic limit of the Hamiltonian in equation (10), we write $B=B_{\mu} d z^{\mu}+B_{\bar{\mu}} d \bar{z}^{\mu}$, replace $z$ by $z / \sqrt{2 I}, \vec{y}$ by $\vec{y} / \sqrt{2 I}$ and $R^{2}$ by $2 I$ and observe that as $I \rightarrow \infty$,

$$
B_{\mu} / \sqrt{2 I} \rightarrow-\frac{1}{2} \bar{z}^{\mu} \quad B_{\bar{\mu}} / \sqrt{2 I} \rightarrow \frac{1}{2} z^{\mu}
$$

as operators, and

$$
h_{i j} \rightarrow \delta_{i j}
$$

therefore,

$$
\hat{H} \rightarrow \hat{H}_{\infty}=\sum_{1 \leqslant \mu \leqslant n}-\frac{1}{M}\left(\nabla_{\mu} \nabla_{\bar{\mu}}+\nabla_{\bar{\mu}} \nabla_{\mu}\right)
$$

where $\nabla_{\mu}=\partial_{\mu}-\frac{1}{2} \overline{z^{\mu}}$ and $\nabla_{\bar{\mu}}=\partial_{\bar{\mu}}+\frac{1}{2} z^{\mu}$. In other words, the Hamiltonian for a single charged particle in QHE on $2 n$ space is

$$
\hat{H}_{\infty}=\sum_{1 \leqslant \mu \leqslant n}\left(-\nabla_{\mu} \nabla_{\bar{\mu}}+\frac{1}{2}\right) \hbar \omega_{c} .
$$

Roughly speaking, equation (15) says that $\hat{H}_{\infty}$ is just the sum of $n$ copies of the Hamiltonian for a single particle in QHE on two-space.

Main results ${ }^{3}$ for QHE on even-dimensional spaces. Let $\hat{h}$ be the Hamiltonian of a single particle in QHE on two-space in the Landau gauge, $V$ be the Bargmann-Fock space [15] of

3 Before taking the limit, the physics is about a charged particle on a sphere under the influence of a natural background gauge field. However, the limit does not have this kind of interpretation any longer. In the semiclassical picture, a $2 n$-dimensional QHE droplet is a finite ball in the configuration space (not $\mathbb{R}^{2 n}$ ) whose Landau levels up to the boundary are all filled. This is clear from our explicit description of the ground-state wavefunctions. 
holomorphic functions on $\mathbb{C}$. Then the Hamiltonian of a single particle in QHE on four-space (in the Landau gauge) is

$$
\hat{H}_{\infty}=\hat{h} \otimes I \otimes I+I \otimes \hat{h} \otimes I
$$

and it acts on the Hilbert space $L^{2}\left(\mathbb{R}^{2}\right) \otimes L^{2}\left(\mathbb{R}^{2}\right) \otimes V$ (here $I$ is the identity operator). The spectrum of this Hamiltonian is

$$
E(q)=(q+1) \hbar \omega_{c}
$$

where $q=0,1,2, \ldots$ and the Hilbert space of ground states is the Bargmann-Fock space of holomorphic functions on $\mathbb{C}^{3}$ with orthonormal basis (when we take the magnetic length as 1)

$$
\psi_{\mathbf{k}}(z)=\frac{z^{[\mathbf{k}]}}{\sqrt{\pi^{3} \mathbf{k} !}} \exp \left(-\frac{1}{2}|z|^{2}\right)
$$

where $\mathbf{k} \equiv\left(k_{1}, k_{2}, k_{3}\right) \in \mathbb{Z}_{+}^{3},|z|^{2} \equiv\left|z_{1}\right|^{2}+\left|z_{2}\right|^{2}+\left|z_{3}\right|^{2}, z^{[\mathbf{k}]} \equiv z_{1}^{k_{1}} z_{2}^{k_{2}} z_{3}^{k_{3}}$ and $\mathbf{k} ! \equiv k_{1} ! k_{2} ! k_{3} !$.

A similar conclusion also holds for QHE on $2 n$ space with $n>2$. In particular, the spectrum of the Hamiltonian is

$$
E(q)=\left(q+\frac{n}{2}\right) \hbar \omega_{c}
$$

where $q=0,1,2, \ldots$ and the Hilbert space of ground states is the Bargmann-Fock space of holomorphic functions on $\mathbb{C}^{\frac{n(n+1)}{2}}$, the configuration space.

One may wonder whether the spectrum and the ground-state wavefunctions for a single particle in QHE on $2 n$ space would be the same if they are obtained as the thermodynamic limit of their counterparts in QHE on $2 n$ spheres. The answer is yes. The spectrum is seen to be the same by comparing equation (19) with equation (6), and the ground-state wavefunctions can be seen to be the same by doing a little further work (see appendix B).

\section{Conclusion}

The recent generalization of the QHE to four-space has been viewed as a significant attempt to answer fundamental questions in physics [11], and it has attracted considerable attention from physicists. In this paper, a clean geometric construction for this generalization is presented; and it actually yields a sequence of models of the QHE type:

QHE on 2 space, Zhang-Hu model, QHE on 6 space, QHE on 8 space 4 ,...

Contrary to the accounts in $[1,11]$, the existence of quantum Hall effects does not crucially depend on the existence of division algebras.

Moreover, the Hamiltonian and the ground-state wavefunctions for a single particle on flat space are derived and explicitly described. The simplicity of this description immediately tells us that QHE on higher dimensional flat space shares common features such as incompressibility with QHE on two-space. We hope the simplicity of this description can also solve some mysteries surrounding QHE on higher dimensional spaces and thus facilitate the search for the grand unification based on QHE on four-space [11].

\footnotetext{
${ }^{4}$ In dimension $8 k$, if we use a real chiral spinor in our construction, the configuration space will be smaller, its dimension will be reduced from $8 k+4 k(4 k-1)=4 k(4 k+1)$ to $8 k+\frac{4 k(4 k-1)}{2}=2 k(4 k+3)$, i.e., from 20 to 14
} when $k=1$. Compare with [1]. 


\section{Acknowledgments}

I would like to thank the referees and the Board Member for their helpful suggestions, and J S Li, X R Wang and S C Zhang for helpful discussions. Especially, I would like to thank Y S Wu for very helpful and very constructive discussions on the physical meaning of the main result of this paper. This work is supported by the Hong Kong Research Grants Council under the RGC project HKUST6161/97P.

\section{Appendix A. Quantum mechanics of a charged particle on homogeneous spaces}

The quantum mechanics of a charged particle on homogeneous spaces has been discussed in many recent papers in mathematical physics $[17,18]$ from more algebraic or computational points of view. Here we give a short presentation of it in geometric language. No originality is claimed because the construction is tautological and obvious to a modern geometer and has already appeared explicitly in [16] (at least) in the special case.

\section{Appendix A.1. Generality}

Let $(\mathrm{X}, h)$ be a Riemannian manifold with Riemannian metric $h$. Consider the quantum mechanics of a neutral particle of mass $M$ freely moving in X; it is well known that the Hamiltonian operator is

$$
\hat{H}=\frac{\hbar^{2}}{2 M} \Delta
$$

where $\Delta$ is the semipositive definite Laplace operator. (In flat Euclidean space, $\Delta=-\sum_{i} \partial_{i}^{2}$.) The Hilbert space of this quantum mechanics problem is the space of square integrable complex-valued functions on X. Following Hodge, we write

$$
\Delta=d^{\dagger} d
$$

where $d$ is the exterior differential operator on complex-valued functions and $d^{\dagger}$ is its formal adjoint. In a local coordinate system, we have

$$
\Delta=-\frac{1}{\sqrt{h}} \partial_{i}\left(h^{i j} \sqrt{h} \partial_{j}\right)
$$

where $h=\operatorname{det}\left(h_{i j}\right)$.

Next we assume the particle is charged, and there is a background gauge field. Geometrically, a background gauge field is just a connection $A$ on a certain Hermitian vector bundle $\xi$ on $\mathrm{X}$. A connection $A$ is equivalent to a linear operator

$$
d_{A}: \Gamma(\xi) \rightarrow \Gamma\left(T^{*} X \otimes \xi\right)
$$

satisfying the Leibnitz rule: $d_{A}(f s)=d f \otimes s+f d_{A} s$, where $s$ is a section of $\xi$ and $f$ is a function on $\mathrm{X}$. The Riemannian metric on $\mathrm{X}$ together with the Hermitian metric on $\xi$ makes possible the definition of the formal adjoint of $d_{A}$ (denoted by $d_{A}^{\dagger}$ ). The obvious generalization of $\Delta$ in equation (2) is

$$
\Delta_{A}=d_{A}^{\dagger} d_{A} .
$$

In a local coordinate system, with a choice of a gauge, we have

$$
\Delta_{A}=-\frac{1}{\sqrt{h}}\left(\partial_{j}+A_{j}\right)\left(h^{j k} \sqrt{h}\left(\partial_{k}+A_{k}\right)\right)
$$


where $A_{i} d x^{i}$ is the Lie algebra-valued one-form representing the connection $A$ in the fixed gauge.

The obvious generalization of equation (1) is

$$
\hat{H}=\frac{\hbar^{2}}{2 M} \Delta_{A} \text {. }
$$

For a general $(\mathrm{X}, h)$ and a general $(\xi, A)$, the quantum mechanics problem is difficult to solve. However, for homogeneous space $\mathrm{X}$ and the associated canonical $(\xi, A)$, the quantum mechanics problem is exactly soluble.

\section{Appendix A.2. Quantum mechanics on homogeneous space}

Let $\mathrm{G}$ be a reductive Lie group, and $\mathrm{H}$ be a compact Lie subgroup. The Cartan-Killing metric on $\mathrm{G}$ gives rise to a canonical Riemannian metric on the homogeneous space $\mathrm{G} / \mathrm{H}$. The principal $\mathrm{H}$ bundle

$$
\mathrm{H} \rightarrow \mathrm{G} \rightarrow \mathrm{G} / \mathrm{H}
$$

has a canonical connection:

$$
A(g)=\operatorname{Pr}_{\mathfrak{h}}\left(g^{-1} d g\right)
$$

where $P r_{\mathfrak{h}}$ is the orthogonal projection onto $\mathfrak{h}$ (the Lie algebra of $\mathrm{H}$ ) using the Cartan-Killing metric on $\mathfrak{g}$ (the Lie algebra of $G$ ).

Let $\mathrm{V}$ be an irreducible unitary representation of $\mathrm{H}$, forming the vector bundle

$$
\xi_{V}: \mathrm{G} \times_{\mathrm{H}} \mathrm{V} \rightarrow \mathrm{G} / \mathrm{H}
$$

The quantum mechanics problem discussed in a previous subsection, when applied in this setting, is completely soluble. It turns out that the problem can be fully described in terms of the representation theory of Lie groups: the Hilbert space, being the square integrable sections of $\xi_{V}$, is called the induced representation of $\mathrm{G}$ (induced from V), and the Hamiltonian is

$$
\hat{H}=\frac{\hbar^{2}}{2 M}\left(c_{2}(\mathrm{G})-c_{2}(\mathrm{H}, \mathrm{V})\right)
$$

where $c_{2}(\mathrm{G})$ is the quadratic Casimir operator of $\mathrm{G}$ and $c_{2}(\mathrm{H}, \mathrm{V})$ is the value of the quadratic Casimir operator of $\mathrm{H}$ on $V$. Here we have used the equation

$$
d_{A}^{\dagger} d_{A}=c_{2}(\mathrm{G})-c_{2}(\mathrm{H}, \mathrm{V})
$$

The proof of this equation is a simple exercise. The key observation is that both sides commute with the induced left action by $\mathrm{G}$ on a space of the $\mathrm{H}$-equivariant map from $\mathrm{G}$ to $\mathrm{V}$. (Note that a section of $\xi_{\mathrm{V}}$ is just an H-equivariant map from $\mathrm{G}$ to V.) Based on this observation, we just need to check that $d_{A}^{*} d_{A} \phi=\left(c_{2}(\mathrm{G})-c_{2}(\mathrm{H}, \mathrm{V})\right) \phi$ at the identity $e$ of G. Next we choose an orthonormal basis $\left\{X_{i}\right\}$ at $T_{e} \mathrm{G}$, such that the first $p$ of the basis vectors forms an orthonormal basis for the orthogonal complement $P$ of $T_{e} \mathrm{H}$ in $T_{e} \mathrm{G}$, so $P \cong \mathbb{R}^{p}$. Locally around $e \mathrm{H}, \mathrm{G} / \mathrm{H}$ is diffeomorphic to $P$ under the exponential map, so it is also diffeomorphic to $\mathbb{R}^{p}$, and this defines a local coordinate map. The next observation is that these local coordinates are geodesic normal coordinates at $e \mathrm{H}$. Then, using the definition of covariant derivatives, we have $\left.d_{A}^{*} d_{A} \phi\right|_{e}=\left.\sum_{i=1}^{p} X_{i} X_{i} \phi\right|_{e}=\left.c_{2}(\mathrm{G}) \phi\right|_{e}-\left.c_{2}(\mathrm{H}) \phi\right|_{e}$. The proof is completed by observing that $\left.c_{2}(\mathrm{H}) \phi\right|_{e}=c_{2}(\mathrm{H}) \cdot \phi(e)$ (here $\cdot$ is the action of $\mathrm{Uh}$ on $\mathrm{V}$ ).

Remark that the Hamiltonian discussed in $[17,18]$ is

$$
\hat{H}^{\prime}=c_{2}(\mathrm{G})
$$

and the one we use here appears explicitly in [16] in the case $\mathrm{G}=S U(2)$ and $\mathrm{H}=U(1)$. 
Appendix B. Concrete description of wavefunctions of a single particle in QHE on even spheres

The main purpose here is to describe the wavefunctions of a single particle in QHE on even spheres at a fixed energy level as certain polynomials on the decompactified configuration space of QHE on even spheres.

For this purpose, we choose the Landau gauge on $S^{2 n} \backslash\{S\}$ and identify $S^{2 n} \backslash\{S\}$ with $\mathbb{C}^{n}$ under the standard stereographical projection map. Also, for each $k \geqslant 1$ we let $Z_{k}$ denote the space of complex $k \times k$ matrices that are skew-symmetric about the second diagonal, and identify $\mathbb{C}^{k} \times Z_{k}$ with $Z_{k+1}$ via

$$
\left(z, z_{k}\right) \mapsto Z \equiv\left(\begin{array}{cc}
z & z_{k} \\
0 & -\tilde{z}
\end{array}\right) .
$$

Here, for $z=\left(z_{1}, \ldots, z_{k}\right)^{T}$ (a column vector), we use $\tilde{z}$ to denote $\left(z_{k}, \ldots, z_{1}\right)$ (a row vector).

Note that $\Delta_{I}^{+}$has a concrete realization as the space of certain polynomials on $Z_{n}$ [19]. Then a smooth section of $\xi_{I}$, being uniquely specified by a smooth map from $\mathbb{C}^{n}$ to $\Delta_{I}^{+}$, can be realized as a smooth map from $\mathbb{C}^{n} \times Z_{n}$ to $\mathbb{C}$ or a smooth map from $Z_{n+1}$ to $\mathbb{C}$.

Therefore, $\mathcal{H}_{I}(q)$ can be viewed as the space of certain smooth maps from $Z_{n+1}$ to $\mathbb{C}$ in a natural way. On the other hand, $\mathcal{H}_{I}(q) \cong \Delta_{I}(q)$-the irreducible representation space of $\operatorname{spin}(2 n+1)$ labelled by the highest weight state $|(q+I) \underbrace{I \cdots I}_{n-1}\rangle$. Since $\Delta_{I}(q)$ has a concrete realization as the space of certain polynomials on $Z_{n+1}$ [19], the description of $\mathcal{H}_{I}(q)$ would be complete if we know the natural identification $\mathcal{H}_{I}(q) \cong \Delta_{I}(q)$. For this purpose, we define a self-diffeomorphism $\phi$ of $Z_{n+1}$ : for $Z=\left(\begin{array}{cc}z & z_{n} \\ 0 & -z\end{array}\right)$, we have

$$
\phi(Z)=\left(\begin{array}{cc}
z & \left(I_{n}+z z^{\dagger}\right) z_{n}\left(I_{n}+\tilde{z}^{\dagger} \tilde{z}\right) \\
0 & -\tilde{z}
\end{array}\right)
$$

where $I_{n}$ is the $n \times n$ identity matrix, and $\dagger$ means Hermitian conjugation. We are now ready to state our conclusion: the one-to-one correspondence $\mathcal{H}_{I}(q) \ni \psi_{f} \leftrightarrow f \in \Delta_{I}(q)$ is

$$
\psi_{f}(Z)=f(\phi(Z))
$$

Moreover, the integration measure used in defining the inner product on the space of wavefunctions is

$$
\mathrm{d} \mu_{I}=\frac{N_{I}(n) \mathrm{d} Z}{\left(1+|z|^{2}\right)^{2 I+2 n}\left|\operatorname{det}\left(I_{n}+z_{n} z_{n}^{\dagger}\right)\right|^{I+(n-1)}}
$$

where $N_{I}(n)$ is the normalization constant chosen such that $\int_{Z_{n+1}} \mathrm{~d} \mu_{I}=1$.

Upon replacing $Z$ by $Z / \sqrt{2 I}$ and taking the limit $I \rightarrow \infty$, we have

$$
\mathrm{d} \mu_{I} \rightarrow \mathrm{d} \mu_{\infty}=\pi^{-\frac{n(n+1)}{2}} \mathrm{e}^{-|Z|^{2}} \mathrm{~d} Z
$$

i.e., the limit of the integration measure is the integration measure of Bargmann-Fock space [15]. It is then easy to see that as $I \rightarrow \infty, \Delta_{I}(0)$ approaches a subspace of the Bargmann-Fock space of holomorphic functions on $\mathbb{C}^{n(n+1) / 2}$, and this subspace is the whole Bargmann-Fock space in the case $n=1$ and $n=2$. An induction argument actually shows that this subspace is the whole Bargmann-Fock space also in the general case.

\section{References}

[1] Bernevig B A, Hu J P, Toumbas N and Zhang S C 2003 The eight dimensional quantum Hall effect and the octonions Preprint cond-mat/0306045 
[2] Chong Y D and Laughlin R B 2003 Metallic nature of the four-dimensional quantum Hall edge Preprint cond-mat/0305697

[3] Sparling G 2002 Twistor theory and the four-dimensional quantum Hall effect of Zhang and Hu Preprint cond-mat/0211679

[4] Chen Y-X 2002 Quasiparticle excitations and hierarchies of 4-dimensional quantum Hall fluid states in the matrix models Preprint hep-th/0210059

Chen Y-X 2002 Matrix models of 4-dimensional quantum Hall fluids Preprint hep-th/0209182

[5] Elvang H and Polchinski J 2002 The quantum Hall effect on $\mathbb{R}^{4}$ Preprint hep-th/0209104

[6] Bernevig B A, Chern C-H, Hu J-P, Toumbas N and Zhang S-C 2002 Ann. Phys., NY 300185 (Preprint cond-mat/0206164)

[7] Zhang S C and Hu J P 2001 Collective excitations at the boundary of a 4D quantum Hall droplet Preprint cond-mat/0112432

[8] Karabali D and Nair V P 2002 Nucl. Phys. B 641 533-46 (Preprint hep-th/0203264)

[9] Fabinger M 2002 J. High Energy Phys. JHEP05(2002)037 (Preprint hep-th/0201016)

[10] Chen Y X, Hou B-Y and Hou B-Y 2002 Nucl. Phys. B 638 220-42 (Preprint hep-th/0203095)

[11] Zhang S C and Hu J P 2001 Science 294823 (Preprint cond-mat/0110572)

[12] Haldane F D M 1983 Phys. Rev. Lett. 51605

[13] Yang C N 1978 J. Math. Phys. 19320

[14] Ramgoolam S 2001 On spherical harmonics for fuzzy spheres in diverse dimensions Nucl. Phys. 610461 (Preprint hep-th/0105006)

[15] Bargmann V 1962 On the representation of the rotation group Rev. Mod. Phys. 34829

[16] Stone M 1989 Supersymmetry and the quantum mechanics of spin Nucl. Phys. 314557

[17] Landsman N P 1992 Induced representations, gauge fields, and quantization on homogeneous spaces Rev. Math. Phys. 4503

[18] McMullan D and Tsutsui I 1996 The canonical connection in quantum mechanics J. Math. Phys. 37625 (Preprint hep-th/9504116)

[19] Zelobenko D P 1973 Compact Lie Groups and Their Representations (Translations of Mathematical Monographs 40) (Providence, RI: American Mathematical Society) 